\title{
Pedicle Periosteum as a Barrier for Guided Bone Regeneration in the Rabbit Frontal Bone
}

\author{
AKIRA HASUIKE ${ }^{1,2}$, HISASHI UJIIE ${ }^{1,3}$, MOTOKI SENOO $^{4}$, MITSUAKI FURUHATA $^{4}$, \\ MAMORU KISHIDA ${ }^{1,3}$, HIDEYASU AKUTAGAWA ${ }^{1,3}$ and SHUICHI SATO ${ }^{1,2}$ \\ ${ }^{1}$ Department of Periodontology, Nihon University School of Dentistry, Tokyo, Japan; \\ ${ }^{2}$ Dental Research Center, Nihon University School of Dentistry, Tokyo, Japan; \\ ${ }^{3}$ Private Practice, Tokyo, Japan; \\ ${ }^{4}$ Division of Applied Oral Sciences, Nihon University Graduate School of Dentistry, Tokyo, Japan
}

\begin{abstract}
Background/Aim: For alveolar ridge reconstruction prior to dental implant placement, a barrier membrane is placed to create space over the bone defect. Although periosteum possesses osteogenic capacity, direct contact between defects and periosteum has been avoided. The present study aimed to investigate whether pedicle periosteum could be used as a barrier membrane. Materials and Methods: Twelve rabbits were used. A U-shaped incision was made in the frontal bone, and the skin-periosteum over the frontal bone was stripped. Two trephine-drilled holes with a diameter of $5 \mathrm{~mm}$ were prepared in the frontal bone. One hole was covered with pedicle periosteum (periosteum side), and the periosteum was secured to the contralateral side. The other defect was covered with an occlusive membrane (membrane side). Results: The histological observation showed that both defects, which were covered either by the periosteum or by the membrane, were closed almost completely after 12 weeks of healing. No statistically significant difference was observed in the bone defect closure rates between the two sides at 4 and 12 weeks. Conclusion: This study demonstrated that the pedicle periosteum possesses regenerative effects equivalent to those of occlusive membrane. The periosteum contributes to new bone formation by acting as a mechanical barrier and a source of osteogenic components.
\end{abstract}

This article is freely accessible online.

Correspondence to: Akira Hasuike, Department of Periodontology, Nihon University School of Dentistry, 1-8-13 Kanda-Surugadai, Chiyoda-ku, Tokyo 101-8310, Japan. Tel: +81 332198349, e-mail: hasuike.akira@nihon-u.ac.jp

Key Words: Alveolar ridge augmentation, bone regeneration, dental implants, skull, X-ray microtomography.
Guided bone regeneration (GBR) is the best documented technique for the treatment of localized bone defects in the jaws (1). GBR has allowed the use of dental implants in the jaw areas with insufficient bone volume. By covering bone defects using occlusive mechanical barriers, such as biodegradable collagen, biodegradable synthetic polymer or non-resorbable polyterafluoroethylene (PTFE) membranes, the invasion of gingival connective tissue and mucosal epithelial cells can be prevented, and the inflow of osteogenic and undifferentiated cells can be retained (2). Given the first successful GBR procedure and the subsequent wide and successful applications of the, PTFE membranes these have become a standard material for bone regeneration (3). This material is resistant to degradation by the host tissues and microbes and it does not elicit immunological reactions.

The periosteum is a tissue that covers bone surfaces and consists of two layers: a superficial layer of collagen fibers and fibroblasts that maintains its mechanical stability, and a deeper, cambium layer of high cellular density (osteogenic layer) that consists of multipotent mesenchymal stem cells, osteoprogenitor cells, and blood vessels (4). Several studies have elucidated the role of periosteum in bone formation. For example, it has been shown that the periosteum can induce bone formation when grafted in ectopic areas (5). Furthermore, bone areas without the periosteum have been found to be strongly compromised in contrast to the areas with an intact periosteum (6). These effects could be explained by the role of periosteum as a potential source of osteogenic cells, growth factors, or even blood. However, since commercial GBR membranes have been designed to be occlusive enough to exclude soft tissue ingrowth (7), these block the inflow of the endogenous resources.

The principles of GBR have been initially applied in the assessment of the region of implantation in fully atrophic jaws (8). The expansion of GBR in a large variety of bone defects has enabled the widespread use of this technique in the clinical practice, and various types of GBR surgical 
techniques have been documented (9-11). There are, however, some cases, where the use of barrier membranes is not warranted for GBR. A current systematic review examining the clinical value of membranes for bone augmentation has concluded that there is insufficient evidence regarding the effects of membranes on bone augmentation procedures to support definitive benefits from their use (12). There is an increasing trend in using titanium meshes as an alternative to membranes for creating the necessary space important for GBR (13). In such cases, the periosteum may play a pivotal role both as a mechanical barrier and a source of blood and osteogenic components.

Although the role of periosteum in bone formation has been elucidated, direct contact between defects and periosteum is usually avoided. There are limited data on the use of periosteum as a GBR membrane compared to the occlusive GBR membranes. The aim of the present study was to investigate whether the pedicle periosteum could be used as a barrier membrane for regeneration of the rabbit frontal bone.

\section{Materials and Methods}

Animals. This study was approved by the Animal Experimentation Committee of the Nihon University School of Dentistry (K05-19). Twelve adult male Japanese rabbits (weight: 2,500-3,000 g) with no injuries or congenital defects were used. All animals were housed in a standard cage, in an experimental animal room (temperature: $22^{\circ} \mathrm{C}$, humidity: $55 \%$, light/dark cycle: $12 / 12$ hours), and were fed a standard laboratory diet and water ad libitum.

Surgical procedures. General anesthesia was induced by injecting pentobarbital sodium (Somnopentyl, Schering-Plough, Munich, Germany), at a dose of $30 \mathrm{mg} / \mathrm{kg}$, via the marginal ear vein, and the frontal bone area was shaved and disinfected with $70 \%$ ethanol. An intraperitoneal injection of $0.5 \mathrm{~mL}$ of lidocaine (1:80,000 dilution) (Xylocaine; Astra Zeneca, Osaka, Japan) was administered to control the bleeding and provide additional anesthesia. A U-shaped incision was made in the frontal bone. Only the outer skin was lifted partially with a small sharp elevator to expose the subcutaneous area without damaging the periosteum and its blood vessels. Thereafter, the periosteum was stripped from the frontal bone and was removed separately. Two trephine-drilled holes with a diameter of $5 \mathrm{~mm}$ each were prepared in the frontal bone (Figure 1A). In each rabbit, one defect was drilled in the right anterior part of the frontal bone and the other one in the left posterior part. Drilling was performed at a low speed under abundant saline irrigation. Following the defect preparation, the surgical area was thoroughly rinsed with physiological saline to wash out any bone fragments. The isolated pedicle periosteum layer was stretched and was placed on top of one defect in a way that it covered the defect $2-3 \mathrm{~mm}$ outside its edges. The layer was secured with 4-0 resorbable sutures (Alfresa Pharma CO., Osaka, Japan). The defect on the contralateral side was covered by a PTFE membrane (Gore-Tex regenerative membrane, Gore Medical, Arizona, US) that was fixed with a medical adhesive (Aron Alpha A "Sankyo", Daiichi Sankyo, Tokyo, Japan) (Figure 1B). Following surgery, the skin was closed with 4-0 resorbable
Table I. Bone defect closure rate in the surgically-produced rabbit frontal bone defects. Values are expressed as mean $\pm S D$. $n=6$ at 4 weeks, $n=6$ at 12 weeks. No statistically significant difference was observed between the two sides.

\begin{tabular}{lcc}
\hline Bone defect closure rate $(\%)$ & 4 weeks & 12 weeks \\
\hline Periosteum side & $85.6 \pm 5.5$ & $95.6 \pm 3.5$ \\
Membrane side & $88.6 \pm 8.5$ & $98.6 \pm 1.3$ \\
\hline
\end{tabular}

sutures (Alfresa Pharma CO., Osaka, Japan). The surgery day was designated as day 0 , and observational periods were 4 and 12 weeks. 6 rabbits were euthanized on 4th week, and 6 on 12th week. Euthanasia was conducted by an overdose injection of pentobarbital sodium (Somnopentyl, Schering-Plough, Munich, Germany) at a dose of $100 \mathrm{mg} / \mathrm{kg}$.

Histological analysis. Following sacrifice, the frontal bones of the rabbits were dissected, fixed with $10 \%$ neutral-buffered formalin, dehydrated, and were embedded in wax. Using a cutting and grinding technique, three $20 \mu \mathrm{m}$-thick histological sections were prepared from the middle of each defect through the longitudinal axis of the bone block. The sections were stained with hematoxylineosin.

Histological examination and morphometric measurements of the hematoxylin-eosin stained sections were performed under a light microscope (Opto-photo-2 light microscope, Nikon, Tokyo, Japan). The bone defect closure rate was determined by measuring the distance between the two defect margins and was expressed as a percentage of the width of the original bone defect. Mann-Whitney $U$-test was used to analyze the differences in bone defect closure rates between the periosteum side and membrane side. A $p$-value of $<0.05$ was considered as statistically significant.

\section{Results}

The healing progressed well in all animals, and no complications or signs of postoperative infection were observed. New bone formation was observed on both defect sides after 4 weeks of healing (Figures 2 and 3). On the periosteum side, the width of the newly-formed bone bridge differed in parts of the defect. Although the connective tissue widely overlaid the newly-formed bone bridge, it was loose and sparse (Figure 2A). The periosteum consisting of a single layer of flat cells was observed just above the newlyformed bone bridge (Figure 2B). On the membrane side, a newly-formed bone bridge with a uniform width was observed between the membrane and the dura matter. The width of the connective tissue covering the newly-formed bone bridge was relatively thinner compared to that of the contralateral side (Figure 3A). The periosteum attached to the newly-formed bone bridge was multi-layered and consisted of cuboidal cells. The membrane placed on the defect was infiltrated by inflammatory cells and erythrocytes (Figure 3B). 

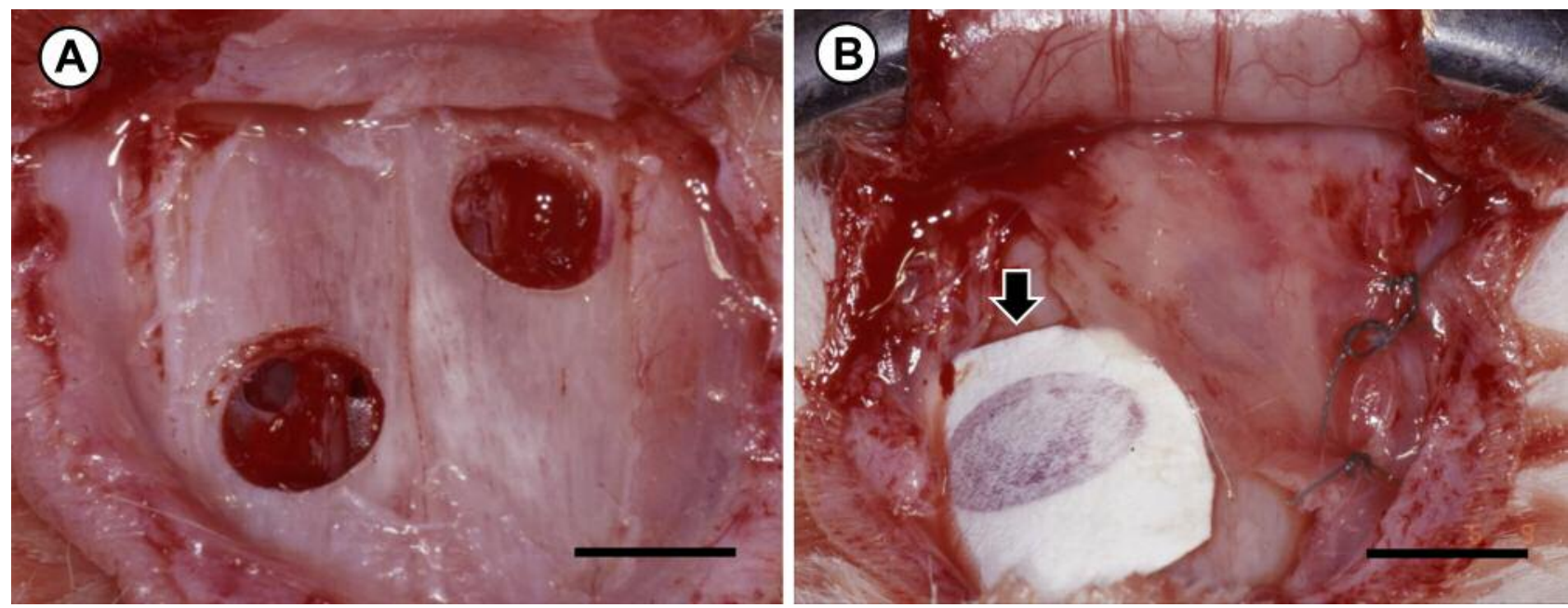

Figure 1. (A) The skin-periosteum was stripped from the frontal bone. Two circular defects were made using a trephine burr. (B) One defect was covered with the periosteum (right side, sutures) and the contralateral defect was covered with the PTFE membrane (left side, arrow). Scale Bars: $5 \mathrm{~mm}$.
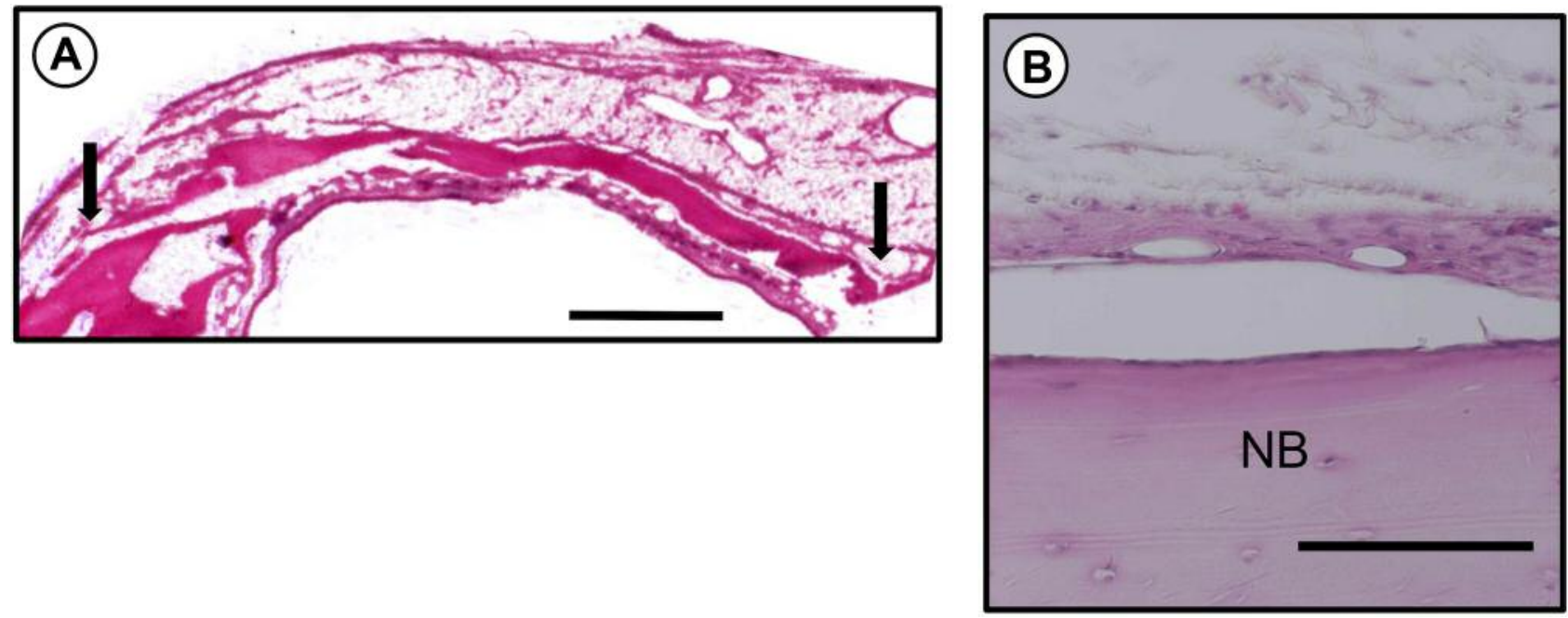

Figure 2. Representative histological observations on the periosteum side after 4 weeks of healing (hematoxylin and eosin staining). (A) Lower magnification (Scale bars: $1 \mathrm{~mm}$ ); (B) Higher magnification (Scale bars: $100 \mu \mathrm{m}$ ). Arrow: Initial edges of the defect; NB: newly-formed bone.

At 12 weeks, all rabbits exhibited almost complete bone healing with newly-formed bone on either side. On the periosteum side, the bony bridge became uniform and thick (Figure 4A). Similarly, the periosteum attached to the bony bridge became thick and multi-layered (Figure 4B). On the membrane side, the uniformly thick bone bridge covered the bone defect (Figure 5A). Multi-layered periosteum was confirmed after 4 weeks of healing, while the infiltration of inflammatory cells and erythrocytes to the membrane disappeared after 12 weeks (Figure 5B).
The bone defect closure rates obtained from the histological sections after 4 and 12 weeks of healing are shown in Table I. In both observational periods, no statistically significant differences were observed between the two sides.

\section{Discussion}

This study examined and compared the effects of pedicle periosteum and GBR membrane on bone regeneration. The histological observation showed that both defects, which 

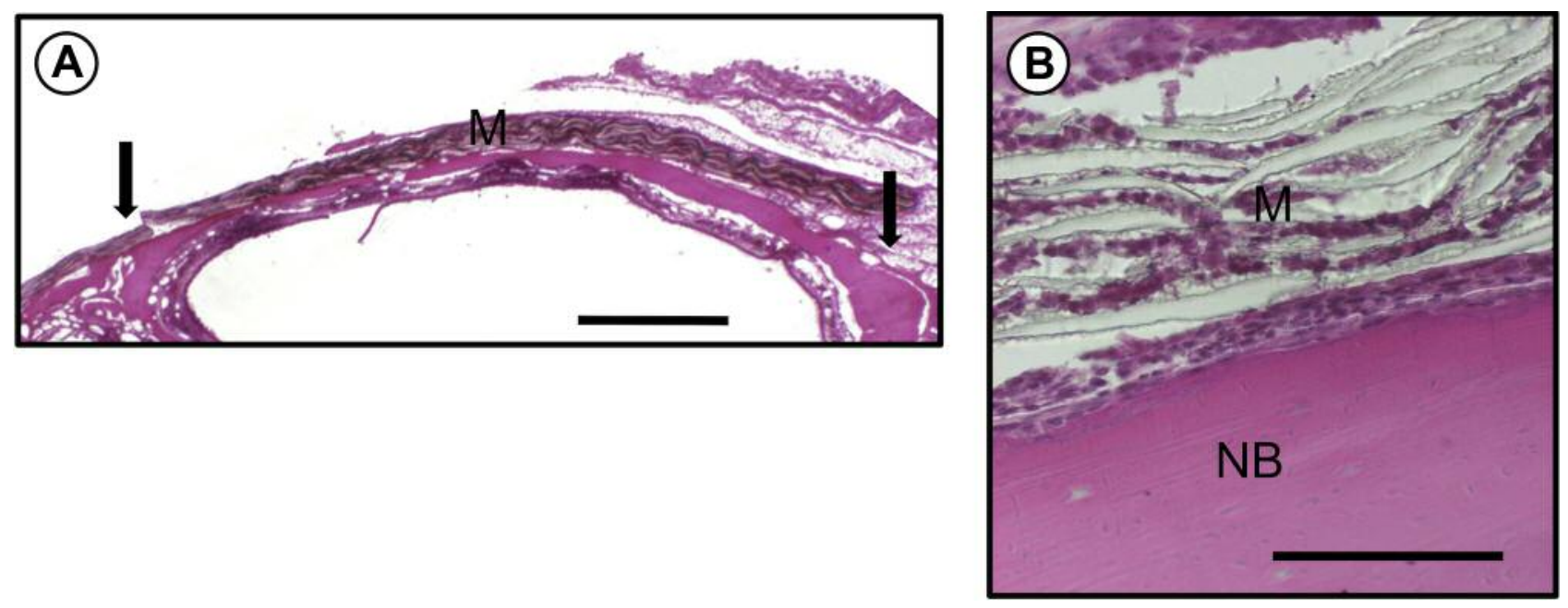

Figure 3. Representative histological observations in the membrane side after 4 weeks of healing (hematoxylin and eosin staining). (A) Lower magnification (Scale bars: $1 \mathrm{~mm}$ ); (B) Higher magnification (Scale bars: 100 um). Arrow: Initial edges of the defect; NB: newly-formed bone; M: membrane.
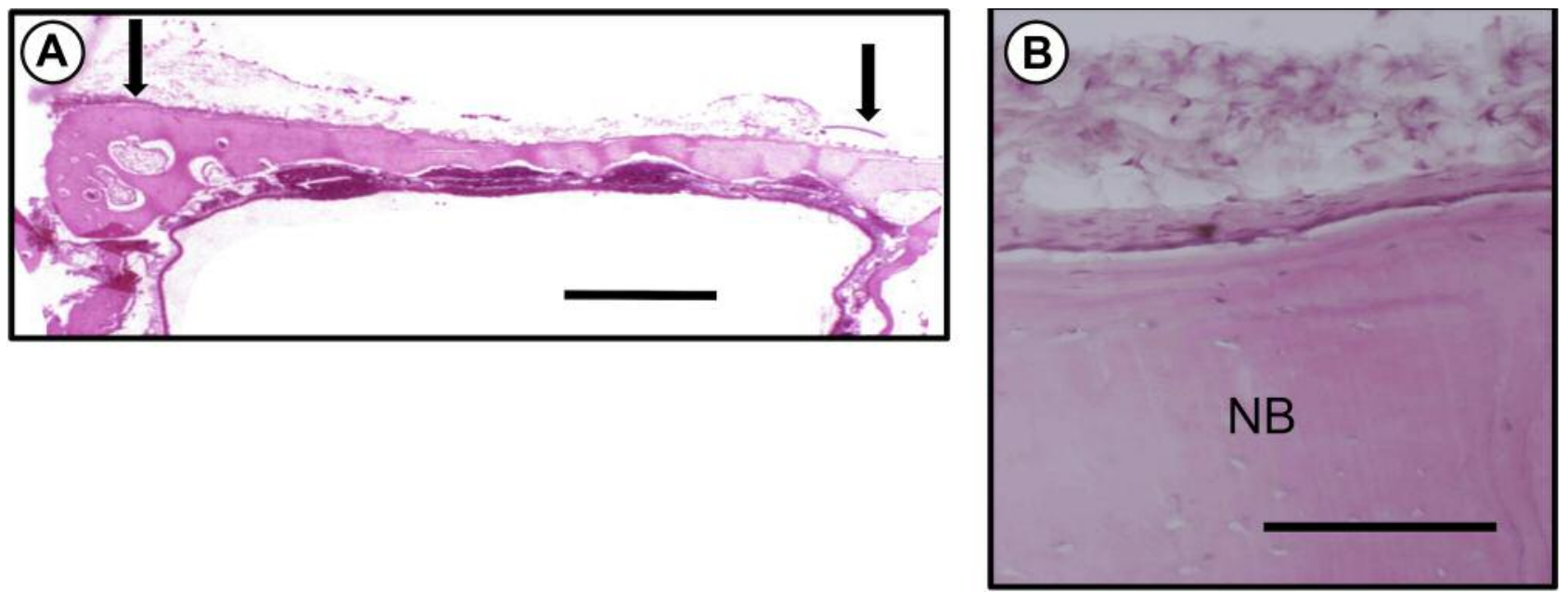

Figure 4. Representative histological observations in the periosteum side after 12 weeks of healing (hematoxylin and eosin staining). (A) Lower magnification (Scale bars: $1 \mathrm{~mm}$ ); (B) Higher magnification (Scale bars: $100 \mu \mathrm{m}$ ). Arrow: Initial edges of the defect: NB: newly-formed bone.

were covered either by the periosteum or by the membrane, were closed almost completely after 12 weeks of healing. These results suggest that the osteogenic capacity of the bone covered by the periosteum does not differ from that of the bone covered by the occlusive membrane.

Fibrous healing, as opposed to osseous regeneration, is a major problem in GBR. The faster-growing epithelium or soft tissue frequently occupies a bone defect, thereby preventing the formation of new bones and causing anatomical and functional abnormalities (14). By using the membrane technique, cells originating from the surrounding bone can repopulate the wound area without any interference from the epithelium or soft tissue (2).

From the present study we can conclude that the pedicle periosteum placed just above the bone defect has osteogenic properties and acts more as a barrier rather than as a resource of fibrous tissue in the rabbit calvaria. Although the periosteum is supposed to have two distinct layers, the composition and thickness of the periosteum differ among animal species and different bone types (4). In the present 

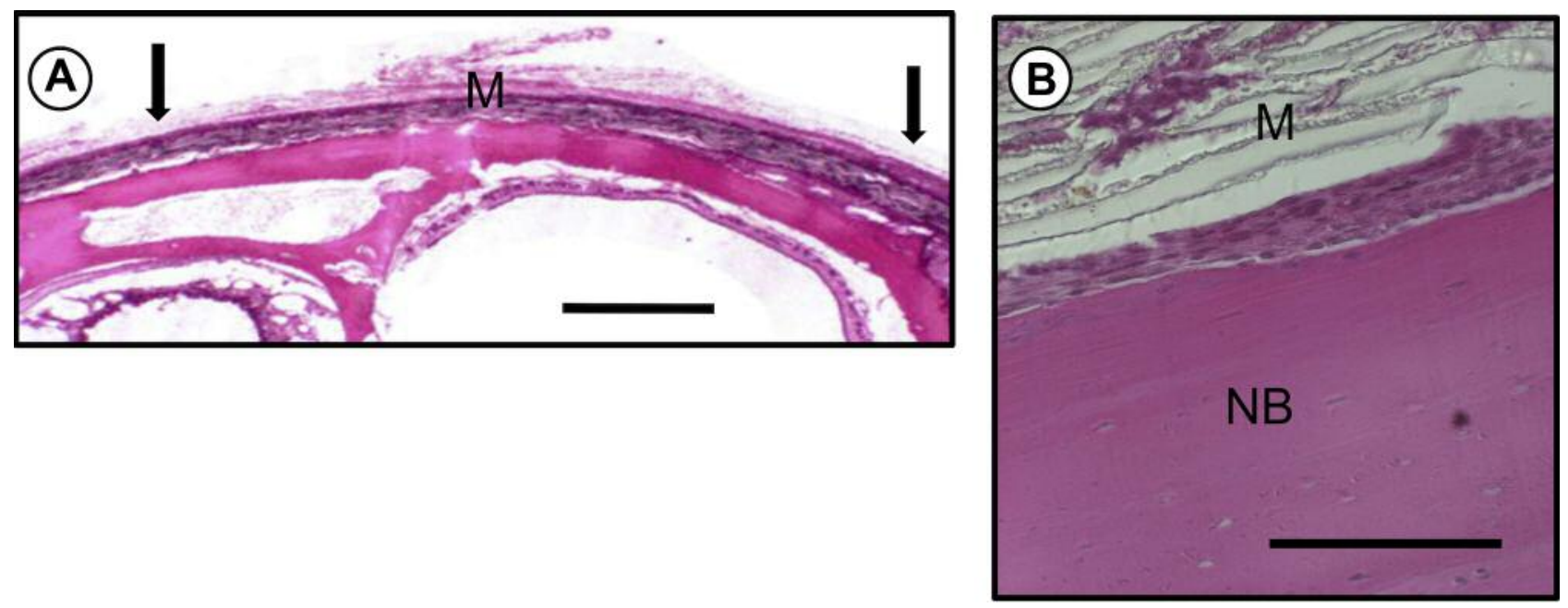

Figure 5. Representative histological observations in the membrane side after 12 weeks of healing (hematoxylin and eosin staining). (A) Lower magnification (Scale bars: $1 \mathrm{~mm}$ ); (B) Higher magnification (Scale bars: $100 \mu \mathrm{m}$ ). Arrow: Initial edges of the defect; NB: newly formed bone; M: membrane.

study, only thin cellar periosteum without thick fibrous tissue was observed in the magnified histological sections of the periosteum side. The periosteum was mobilized and was placed over the defect, with the cambium layer was juxtaposed to the exposed defect. This cell-rich periosteum played a role as a border between the bone and soft tissue, and the sparse fibrous tissue was located on the outer part of the periosteum. This peculiar characteristic of the rabbit calvarial periosteum may be responsible for its role as a mechanical barrier. It should be mentioned that, in the clinical settings, it is important to know the characteristics of the periosteum in the treatment target.

In both observational periods, the bone defect closure rates on the periosteum side were slightly lower compared to the membrane side. In addition, he healed bone in that region was thinner compared to the defects covered by the membrane. This is most likely because the periosteum over the defective rabbit frontal bone was not thick or rigid to provide enough space for bone regeneration. Keeping the space for bone regeneration is one of pivotal factors for the success of GBR. Without rigidity of barrier, membrane could be collapse into space for regeneration, and deteriorate new bone formation. In the case clinicians use periosteum as a barrier membrane, they should use an additional framework. In such cases, titanium mesh could be the best option to create the space for bone formation (15). The titanium mesh rigidity prevents contour collapse, and its elasticity makes it easier to trim and shape accordingly. Thanks to the presence of pores, titanium mesh can adopt a three-dimensional shape without blocking the inflow of osteogenic resources and the blood supply from the periosteum.

Previously, Peltola et al. examined and compared the healing effects of pedicle periosteum flaps and free periosteum grafts using the rabbit front and calvarial bone defects (16). The group demonstrated that the defect covered by the pedicle periosteum flap shows more vigorous new bone formation compared to the free periosteum. In the present study, the attachment of the autogenous periosteal membrane with the mucoperiosteal flap facilitated the maintenance of its vascular supply. This is important for the healing and maintenance of the vital cambium layer, which has the potential to stimulate bone formation. Recently, the application of isolated periosteum cells (17) and periosteal sheets for bone regenerative therapy (18) has been a focus of research. Although these treatments have a high potential, the obtained osteogenic units are supposed to be transplanted as free grafts in both therapies. Thus, blood circulation would be one of the key issues to focus on in these future therapies.

We acknowledge that there are several limitations in this study. First, we assessed the role of pedicle periosteum and occlusive membrane on bone regeneration in a model of noncritical bone defects. When bone substitutes or surgical techniques are assessed for their osteogenic potential, the experimentally prepared bone defect should be large enough to preclude the spontaneous healing. The smallest size of a bone defect that does not heal spontaneously is termed as a critical-size defect, which has been reported to be approximately $6 \mathrm{~mm}$ in the rabbit distal radius, $15 \mathrm{~mm}$ in the calvaria, and $5 \mathrm{~mm}$ in the mandible (19). Further studies are required to determine the critical-size defect in the rabbit frontal bone. Second, the frontal bone of the rabbit was used for the experiments here. As previously stated, the composition and thickness of the periosteum differ among animal species (4), so one would need to determine these specific parameters for this type of bone. Additional studies 
using the jaw bone of non-human primates could of value in the field of GBR. Third, we assessed the regenerative effect of the frontal bone only by histomorphometric approaches. For a detailed analysis of the effects of the periosteum on bone regeneration, additional immunohistological or genetic approaches should also be considered in future studies.

In conclusion, this study demonstrated that the pedicle periosteum possesses regenerative properties equivalent to those of the occlusive membrane. Despite the limitations of the study, we suggest that the periosteum contributes to new bone formation by acting as a mechanical barrier and a source of osteogenic components.

\section{Conflicts of Interest}

The Authors have no conflicts of interest to declare.

\section{Authors' Contributions}

AH wrote the initial draft of the manuscript. HU and SS developed the research concept and design. MS and MF conducted experiment and contributed to analysis and interpretation of data. MK and HA assisted in the preparation of the manuscript. All other authors have contributed to data collection and interpretation, and critically reviewed the manuscript. All authors approved the final version of the manuscript and agreed to be accountable for all aspects of the work in ensuring that questions related to the accuracy or integrity of any part of the work are appropriately investigated and resolved.

\section{Acknowledgements}

This study was supported by JSPS KAKENHI Grant Number 17K11810 and by Shuichi Sato.

\section{References}

1 Benic GI, Hämmerle $\mathrm{CH}$ : Horizontal bone augmentation by means of guided bone regeneration. Periodontol 2000 66: 13-40, 2014. PMID: 25123759. DOI: 10.1111/prd.12039

2 Elgali I, Omar O, Dahlin C and Thomsen P: Guided bone regeneration: materials and biological mechanisms revisited. Eur J Oral Sci 125: 315-337, 2017. PMID: 28833567. DOI: 10.1111/ eos.12364.

3 Dahlin C, Linde A, Gottlow J and Nyman S: Healing of bone defects by guided tissue regeneration. Plast Reconstr Surg 81 : 672-676, 1988. PMID: 3362985.

4 Lin Z, Fateh A, Salem DM and Intini G: Periosteum: biology and applications in craniofacial bone regeneration. J Dent Res 93: 109116, 2014. PMID: 24088412. DOI: $10.1177 / 0022034513506445$

5 Cohen $J$ and Lacroix P: Bone and cartilage formation by periosteum. Assay of experimental autogenous grafts. J Bone Joint Surg Am 37-A: 717-730, 1955. PMID: 13242605.

6 Gruber R, Stadlinger B and Terheyden H: Cell-to-cell communication in guided bone regeneration: Molecular and cellular mechanisms. Clin Oral Implants Res 28: 1139-1146, 2017. PMID: 27550738. DOI: 10.1111/clr.12929
7 Schmid J, Hämmerle CH, Olah AJ and Lang NP: Membrane permeability is unnecessary for guided generation of new bone. An experimental study in the rabbit. Clin Oral Implants Res 5: 125-30, 1994. PMID: 7827226.

8 Simion M, Jovanovic SA, Trisi P, Scarano A and Piattelli A: Vertical ridge augmentation around dental implants using a membrane technique and autogenous bone or allografts in humans. Int $\mathrm{J}$ Periodontics Restorative Dent 18: 8-23, 1998. PMID: 9558553.

9 Garcia J, Dodge A, Luepke P, Wang HL, Kapila Y and Lin GH: Effect of membrane exposure on guided bone regeneration: A systematic review and meta-analysis. Clin Oral Implants Res 29: 328-338, 2018. PMID:29368353. DOI: 10.1111/clr.13121

10 Plonka AB, Urban IA and Wang HL: Decision Tree for Vertical Ridge Augmentation. Int J Periodontics Restorative Dent 38: 269-275, 2018. PMID: 29447321. DOI: 10.11607/prd.3280

$11 \mathrm{Kim} \mathrm{JM}$, Kim JH, Lee $\mathrm{BH}$ and Choi SH: Vertical bone augmentation using three-dimensionally printed cap in the rat calvarial partial defect. In Vivo 32: 1111-1117, 2018. PMID: 30150433. DOI: 10.21873/invivo.11353

12 Jonker BP, Roeloffs MW, Wolvius EB and Pijpe J: The clinical value of membranes in bone augmentation procedures in oral implantology: A systematic review of randomised controlled trials. Eur J Oral Implantol 9: 335-365, 2016. PMID: 2799050.

13 Assenza B, Piattelli M, Scarano A, Lezzi G, Petrone G and Piattelli A: Localized ridge augmentation using titanium micromesh. J Oral Implantol 27: 287-292, 2001. PMID: 12498436. DOI: 10.1563/15481336(2001)027<0287:LRAUTM > 2.3.CO;2

14 Dimitriou R, Mataliotakis GI, Calori GM and Giannoudis PV: The role of barrier membranes for guided bone regeneration and restoration of large bone defects: current experimental and clinical evidence. BMC Med 10: 81, 2012. PMID: 22834465. DOI: $10.1186 / 1741-7015-10-81$

15 Rasia-dal Polo M, Poli PP, Rancitelli D, Beretta M and Maiorana C: Alveolar ridge reconstruction with titanium meshes: a systematic review of the literature. Med Oral Patol Oral Cir Bucal 19: e639-46, 2014. PMID: 25350597.

16 Peltola MJ, Aitasalo KMJ, Suonpaa JTK, Yli-Urpo A and Laippala $\mathrm{PJ}$ : In vivo model for frontal sinus and calvarial bone bone defect obliteration with bioactive glass S53P4 and hydroxyapataite. J Biomed Mater Res 58: 261-269, 2001. PMID: 11319739.

17 Ruvalcaba-Paredes EK, Hidalgo-Bastida LA, Sesman-Bernal AL, Garciadiego-Cazares D, Pérez-Dosal MR, Martínez-López V, Vargas-Sandoval B, Pichardo-Bahena R, Ibarra C and Velasquillo C: Osteogenic potential of murine periosteum for critical-size cranial defects. Br J Oral Maxillofac Surg 54: 772777, 2016. PMID: 27282080. DOI: 10.1016/j.bjoms.2016.05.001

18 Horimizu M, Kubota T, Kawase T, Nagata M, Kobayashi M, Okuda K, Nakata K and Yoshie H: Synergistic effects of the combined use of human-cultured periosteal sheets and plateletrich fibrin on bone regeneration: An animal study. Clin Exp Dent Res 3: 134-141, 2017. PMID: 29744191. DOI: 10.1002/cre2.71

19 Spicer PP, Kretlow JD, Young S, Jansen JA, Kasper FK and Mikos AG: Evaluation of bone regeneration using the rat critical size calvarial defect. Nat Protoc 7: 1918-1929, 2012. PMID: 23018195. DOI: $10.1038 /$ nprot.2012.113

Received January 26, 2019

Revised February 15, 2019

Accepted February 19, 2019 